\title{
Diagnostic Dilemma in Metaplastic Chondroid Breast Carcinoma
}

\author{
Farina MYa, Shahrun Niza $A S^{a}$, Saladina JJa, Nani Harlina $M L^{\mathrm{a}}$, Zaireen $M N^{\mathrm{a}}$, Nurismah $M \mathrm{M}^{\mathrm{b}}$, Rohaizak $\mathrm{M}^{\mathrm{a}}$ \\ aEndocrine and breast surgery unit, Department of Surgery, Faculty of Medicine, UKM Medical Centre, Kuala \\ Lumpur, Malaysia \\ 'Department of Pathology, Faculty of Medicine, UKM Medical Centre, Kuala Lumpur, Malaysia
}

\begin{abstract}
Metaplastic breast cancer is a rare form of primary breast cancer. It contains a mixture of adenocarcinoma with metaplastic elements. It is important to differentiate with primary sarcoma of the breast which carries different treatment strategies and prognosis. A 55-year-old lady previously diagnosed to have a left breast cancer in the year 2000 and carcinoma of the endometrium in 2009, presented with a right breast lump. A trucut biopsy reported as an infiltrating ductal carcinoma with background of chondromyxoid and cartilagenous matrix, most probably metaplastic carcinoma. A wide local excision with sentinel lymph node biopsy was performed, and the final histology was consistent with metaplastic chondroid carcinoma of the breast with no evidence of metastsis. The surgery was followed by adjuvant radiotherapy and currently free from any recurrence. The diagnostic dilemma on this very rare condition is reviewed.
\end{abstract}

KEYWORDS: Metaplastic, breast cancer, chondroid, cartilage

\section{INTRODUCTION}

Metaplastic breast carcinomas (MBC) are rare forms of primary breast malignancies. The term refers to a heterogeneous group of tumours characterized by a mixture of adenocarcinoma (i.e. usual types of breast cancer) with metaplastic elements, which can be homologous (squamous or spindle metaplasia) or heterologous (chondroid, osseous or lipomatous differentiation). ${ }^{1} \mathrm{MBC}$ poses a challenge to both pathologist and clinician in distinguishing the distinction between metaplastic breast cancer and sarcoma, which is clinically important because the surgical treatment and radiotherapy and /or chemotherapy of different pathways.

Corresponding author:

Prof Dr Rohaizak Muhammad

Endocrine and breast surgery unit,

Dept. of Surgery,

Faculty of Medicine

UKM Medical Centre,

Jalan Yaakub Latif, Bandar Tun Razak

56000 Cheras, Wilayah Persekutuan Kuala Lumpur

Tel no : +60193122555

Fax No: +60391456684

Email : rohaizak@ppukm.ukm.edu.my

\section{CASE REPORT}

A 55-year-old female was diagnosed to have a left breast cancer in 2000. She underwent a left wide local excision with axillary clearance, followed by adjuvant chemotherapy and radiotherapy. She was also diagnosed to have carcinoma of the endometrium in 2009 and underwent total abdominal hysterectomybilateral salphingo-oophrectomy (TAHBSO).

During the follow up, she was noted to have a right breast lump in early 2012. There was no palpable axillary lymph node. The mammography assessment showed a mass associated with microcalcifications at right upper inner of breast, and the ultrasound showed a well-circumscribed hypoechoic lesion measuring $2.0 \times 1.5 \mathrm{~cm}$ in diameter. A core needle biopsy was performed, and microscopic examination revealed fragments of breast tissue infiltrated by malignant cells with background of a chondromyxoid and cartilaginous matrix. Immunohistochemical stainings were positive for CKAE/AE3 and S100 markers but negative for ER and PR. The Cerb-2 was $1+$. The biopsy specimen was suggestive of infiltrating carcinoma with malignant cartilage component, most probably metaplastic carcinoma. She later underwent a wide local excision of the right breast with axillary sentinel nodes excision.

The histopathology report was consistent with metaplastic carcinoma with chondroid differentiation (Figure 1) and the sentinel node was negative for tumour metastasis. She underwent radiotherapy as part of her subsequent treatments. 


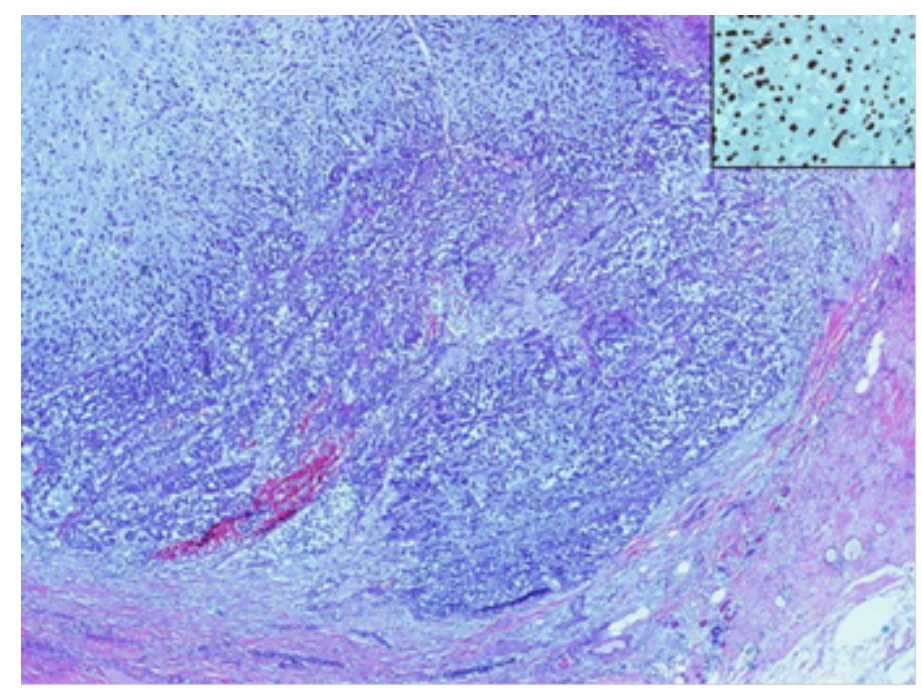

Figure 1. Low power view (x4 magnification) of metaplastic carcinoma with well delineated pushing borders. Areas of chondroid differentiation are seen towards the center of the lesion (left upper). The carcinoma component is seen at the periphery forming tubules. (Stain: Hematoxylin and eosin). Inset: Immunohistochemically, the chondroid components are S-100 positive

\section{DISCUSSION}

Metaplastic breast carcinoma $(M B C)$ is relatively uncommon and accounts for less than $5 \%$ of all mammary tumours. ${ }^{2,3}$ It is a term of a range of cancers of mixed epithelial and mesenchymal component in origin or squamous differentiation. The alternate or historical names are adenosquamous carcinoma, carcinosarcoma, matrix producing carcinoma, sarcomatoid carcinoma, spindle cell carcinoma and squamous-cell carcinoma. Regardless of the name and subtypes, $91 \%$ of $M B C$ displayed a basal like phenotype, which tend to be more aggressive than the luminal IDC. ${ }^{4,5}$

Commonly, the patients presented with a palpable painless breast lump, and further assessment is done in triple assessment manner. $M B C$ has many different histologic features, and specific immunohistochemistry stainings are required for diagnosis. ${ }^{6}$ The aetiology of this type of breast cancer is so far unknown and there are no specific radiological features of $M B C$. It varies from well-defined to ill-defined and spiculated, calcified to non-calcified. ${ }^{3}$ In the case presented, ultrasonographically, a well circumscribed mass was seen and showed microcalcification on the mammogram. Only the trucut biopsy, clinched the diagnosis of metaplastic breast cancer. Hence, the challenge is with regards to screening mammography and confirmatory diagnosis.

Some literature reports of the $M B C$ tumours are relatively large on presentation but often with node negative during examinations. $M B C$ presents with axillary nodal involvement less frequently than adenocarcinma of the breast on which accounts between $6-26 \%$ of the cases. ${ }^{2}$ High suspicious of this condition needed among clinician to ascertain diagnosis and to proceed with appropriate treatment options either breast conserving surgery or mastectomy. The exploration for nodal involvement was limited to palpable lymph nodes and the diagnosis of infiltrating ductal carcinoma as in the case presented. The sentinel lymph nodes biopsy gives an alternative to identify the possibility of nodal metastasis in non-enlarged lymph nodes with minimum morbidity. The Mayo Clinic Foundation has suggested systemic therapy appears to be less effective in MBC. The disease-free survival and the overall survival were found to be decreased compare to typical adenocarcinomas. ${ }^{2}$

In this patient, she was previously treated for left breast cancer and already underwent a course of chemotherapy and radiotherapy after surgery. She developed a different form of lesion on the contralateral breast after 12 years. The histology examination showed a circumscribed tumour with invasive pushing borders composed of mixture of epithelial and chondroid component with immunohistochemistry staining positive for CKAE1/AE3 and negative foe ER, PR and Cerb-B2 at the peripheral of lesion and the chondroid component is positive for $\mathrm{S} 100$ and focally positive for CKAE1/AE2 as reported by Fenyvesi. ${ }^{7}$ The different parts of the lesion were assessed accordingly to identify the types of component involved in this MBC. She underwent treatment as in the same principles as Invasive Ductal Carcinoma.

\section{CONCLUSION}

$M B C$ is a rare tumour with a range of histological characteristics. It is commonly presented with larger tumour size, less nodal involvement and hormone receptor negative. Specific immunohistochemistry staining is essential in the diagnosis and identifying the subtypes of the MBC. The Treatment options are breast 
conserving surgery or mastectomy accordingly. The role of neo-adjuvant / adjuvant chemotherapy and radiotherapy are still unclear. Innovative therapeutic regimes can be offered to the appropriate candidates.

\section{REFERENCES}

1. Barnes PJ, Boutilier R, Chiasson D, Rayson D. Metaplastic breast carcinoma: clinical-pathologic characteristics and HER2/neu expression. Breast Cancer Res Treat 2005; 91:173-8.

2. Rayson D, Adjei AA, Suman VJ, Wold LE, Ingle $J \mathrm{~N}$. Metaplastic breast cancer: prognosis and response to systemic therapy. Ann Oncol 1999; 10:413-9.

3. Greenberg D, Mclntyre H, Bierre T. Metaplastic breast carcinoma. Australas Radiol 2004; 48:2437.

4. Reis-Filho JS, Milanezi F, Steele D, et al. Metaplas tic breast carcinomas are basal-like tumours. His topathology 2006; 49:10-21.

5. Gwin K, Wheeler DT, Bossuyt V, Tavassoli FA. Breast carcinoma with chondroid differentiation: A clinicopathologic study of 21 triple negative (ER- ,PR-,Her2/neu-) cases. Int J Surg Pathol 2010; 18:27-35.

6. Patterson SK, Tworek JA, Roubidoux MA, Helvie MA, Oberman HA. Metaplastic carcinoma of the breast: mammographic appearance with pathologic correlation AJR Am J Roentgenol 1997; 169:709-12

7. Fenyvesi A. A case of carcinoma of the breast with metaplasia to chondrosarcoma: light micro scopic and immunohistochemical features. Archive of Oncology 2003; 11:273-6. 
HE INTERNATIONAL MEDICAL JOURNAL MALAYSIA 